\title{
Assess the use of Solar Dryer with Photonic Solar Reflectors and PCMs in Farming Products in the Andean Region
}

\author{
Estefanía Orquera ${ }^{1}$, Álvaro Aguinaga ${ }^{2}$, Carlos Ávila $^{3}$, Víctor Hidalgo $^{4}$ \\ ${ }^{1,2,3,4}$ Department of Mechanical Engineering/ Escuela Politécnica Nacional \\ Ladrón de Guevara E11-253, Quito, Ecuador \\ estefania.orquera@epn.edu; alvaro.aguinaga@epn.edu.ec, \\ carlos.avila@epn.edu.ec, victor.hidalgo@epn.edu.ec
}

\begin{abstract}
Dehydration food products is seen as an alternative to preserve them in quality and extend their period of live and consumption, this procedure usually happens using non-renewables resources, or in the open air, which, in some cases, could contaminate the final product. An alternative is the use of solar energy for solar dyers to dehydrate agricultural products. Since Ecuador has an excellent radiant resource and an economical dependence of Agriculture activities, it is suggested to assess the performance of a solar dryer, that consider, as an improvement the use of Integrated Photonic Solar Reflectors and thermal energy storage with Phase Change Materials (PCMs) in the Province of Pichincha, in the Andean Region, applied to farming products harvesting by a local community in Cayambe. For this evaluation, in the begging will be analysed food products such as Maize and Cedron leaves, and it will be evaluated the reduction of their moisture content. The use of thermal energy storage (PCMs) will allow to show that this incorporation increase the efficiency of the solar energy system, and the addition of Integrated Photonic Solar Reflectors to the panels, also, it will be possible to suggest improvements in the design of the chosen solar dryer taken as reference for the purposes of this research.
\end{abstract}

Keywords: Solar Dryer, Integrated Photonic Solar Reflectors, PCM, Thermal Energy Storage, Dehydration, Moisture Content, Irradiation

\section{Introduction}

Agriculture in Ecuador is an essential economic activity, which is performed mostly by families in rural areas that economically depend on their farming production. Nevertheless, it is known that around $6 \%$ of global food losses, in the post harvesting process and due to the ripening of organic products, happen in Latin America and in the Caribbean Region and, in the following years this production will be also affected by climatic events such as: drought seasons, stronger winters, extreme temperature changes, which will increase social, economic, and development inequalities. [1] [2] [3]

To reduce these losses and to add value to agricultural products, emerges the idea of dehydrated these items, i.e. drying to preserve them, and for this purpose it is required amounts of energy which could be obtained by nonrenewable and renewable energy sources. In the first case, fossil fuels and carbon sources have environmental constraints due to the $\mathrm{CO}_{2}$ emissions, costs and the ecological impacts of their use; on the other hand, and for this project, it is suggested the employment of solar energy for the dryer, which is a renewable source of energy, it is abundant, and environmental friendly with less impact in the planet. [4]

Solar dryers rely on solar radiation, available in daily hours and in sunny seasons conditions, and their limitation appears during night hours, cloudy or winter seasons, therefore, and for this survey, it is proposed the use of solar dryers, that counts with thermal energy storage such as Phase Change Materials (PCMs) that gathers energy and release it when a change of state occurs (liquid-solid and solid- liquid), and with these elements incorporated in the dryer system, it is expected an increase in the total thermal efficiency. [5] [6]

Several surveys around the world have been conducted about solar dryer for food products, such as the one performed by Bal et al. "Solar dryers with thermal energy storage for agricultural food", "Solar dryer with PCM as Energy storage medium (Review)", however, there is no one conducted in Ecuador in the Andean Region where the solar resource is approx. $5.275 \mathrm{kWh} / \mathrm{m} 2 /$ day. [7] [8] [9]

In this research it is suggested to assess the employment of solar dryer with Integrated Photonic Solar Reflectors and PCMs to dry farming products in the Andean Region. There were chosen Maize (Corn), and Cedron leaves (Aloysia citriodora), the first is considered an essential nourishment in Ecuador, and the Cedron leaves as a medicinal herb has many benefits for the organism and the nervous system, and it is also a South American product. Maize and 
Cedron leaves, as long others are produced in the Andean Region, and specifically in "Casa Campesina Cayambe", a rural community where families farm and sell their own products without middlemen. [10] [11]

\section{Solar Dryer}

The employment of solar energy in a dryer consist in to heat the air that will remove moisture from agricultural food, the moisture content varies depending on the product properties, and to warranty the dehydration process and the quality of the treated product the operational conditions should be controlled. Usually the range of work temperature for dehydration process for some products is around 50 to $60{ }^{\circ} \mathrm{C}$. [7] [12]

Solar dryers could be classified in natural and forced convection dryers, and, according on the assembly of solar panels, the air movement mode, the product to dry, solar dryers could be categorized in:

- Direct solar dryers

- Indirect solar dryers

- Hybrid solar dryers

\subsection{Direct solar dryers}

In this type of solar dryer, the food product is directly heated by the solar radiation, and the moisture is removed by the natural air circulation. [7] [12]

The basic configuration of a direct solar dryers has as benefit to protect the food product from pollution, rain, dust, etc. thanks to its transparent cover, still, the assembly has as constraints the difficulty of control overheating, which will impact in the quality of the product. Nevertheless, this type of solar dryers is the most used for this applications.

\subsection{Indirect solar dryers}

Indirect solar dryers are comprised by a solar air heater, a drying unit, and a fan or blower to duct the air that is heated into the dryer chamber. This configuration can achieve higher temperatures compared to Direct solar dryers, and thanks to the blower, it is possible to control and maintain a desired air flow rate for the drying process, increasing the efficiency of moisture removal in agricultural products. [7] [12]

As examples of the use of indirect solar dryers, there is the study perform by Kumar et all in 2016, which employed this configuration in order to dry mint leaves in Turkey, and, the survey of Bhardwaj et al. in 2017, that uses indirect solar dryers with PCMs to dry a medicinal herb such as valeriana jatamansi. [13] [4]

\subsection{Hybrid solar dryers}

In this type of solar dryer, the food product is heated by a combination of direct solar radiation and the heating air coming from a solar air heater, which usually employs as energy source electricity, biomass, etc. This configuration is nowadays the preferred alternative to dry food products in the fastest way without jeopardize the quality of the product. [7] [12]

As examples, there is the survey conducted by Bena and Fuller [14] which performed a combination of direct solar dryer with a biomass burner to dry agricultural products, and the study perform by Bal et al. [8] to dehydrated aromatic plants, using direct solar dryers. Reyes et al., conducted an study that employs Hybrid solar dryers to dehydrated Mushrooms, and along with this arrangement, the use of PCMs to increase the efficiency of the dryer. [6].

\section{PCMs review}

Solar energy has as constraint the availability only in sunny hours, thermal energy storage is an alternative that could be used to satisfy the lack of energy in periods of time where sun is not available, due to winter seasons, cloudy days or by night. Phase Change Materials (PCMs) could supply the energy demand in required moments, and therefore, increase the efficiency of the solar systems. [6] PCMs are elements that storage "latent" heat, which is expressed as the ability to release energy while changing of state or phase, liquid to solid and solid to liquid. In the market, according to the required temperature range, there are a wide variety of PCMs available, and they are classified as: organic, inorganic and eutectic materials. [15] [16]

\subsection{Organic PCMs}


These type of PCMs are characterized by having in their molecular structure atoms of carbon and hydrogen, and they are classified as paraffin and non-paraffins. A congruent melting occurs in these materials, which is that the melting and freezing happens without a phase segregation and a permanent loss of their latent heat of fusion, in selfnucleation, the elements crystallize with little or no supercooling and usually non-corrosiveness. Paraffin wax, with a costs around \$2/kg is the PCM mainly used in commercial applications. [16] [17]

\subsection{Inorganic PCMs}

Inorganic PCMs are those that in their molecular structure do not have carbon atoms, they are usually water based, known as aqueous, they do not supercool substantially, and their heats of fusion do not decline with cycling. Some of their features are good thermal conductivity and high volumetric energy storage density. Some constrains are leakage and causticity. Usually they are classified as Salt hydrates, the most common one is calcium chloride with a cost of $\$ 0.20 / \mathrm{kg}$, and Metallics. [15] [17]

\subsection{Eutectic PCM}

The eutectic PCMs are those integrated by at least two different components of inorganic salts such as nitrates, sulphates, chlorides salts of metals like magnesium, potassium, lithium or calcium. Each of these components melt and freeze in a harmony way giving as a result a mixture of crystal components during crystallization. Most times, eutectic PCMs melt and freeze without segregation because they freeze to an intimate mixture of crystals leaving a small chance for the constitutive components to separate. During the melting process the components condense at the same time, and for that reason a physical detachment does not occur. [6] [15]

\section{Integrated Photonic Solar Reflectors}

The irradiation that comes from the sun usually is not efficiently received and captured in solar devices, due to, in most of the cases, the energy difference between the absorption of the appliances and the sun's broadband irradiation which represent spectrum losses. Taking in consideration this fact, it is suggested the employment of photon recycling technologies that offer the possibility to convert low solar energy photons into higher energy photons that could improve the efficiency performance of solar power production and solar energy storage.[18]

Some highly absorbing technologies and light trapping cells are available in the market. It is proposed the employment of reflecting layers, nano-layer filters based on silicon dioxide and tantalum dioxide in solar cells, which will enhance the solar irradiation absorption, and therefore, the efficiency of the solar system. As an example, in the survey "Semitransparent Polymer Solar Cells with 5\% Power Conversion Efficiency Using Photonic Crystal Reflector" conducted by Yu et all [19], it is mentioned that the semi-transparent polymer solar cells (ST-PSCs) has added the onedimensional photonic crystals (1DPCs) as a high reflector. This Integrated Photonic Solar Reflectors is composed by several pairs of $\mathrm{WO} 3(65.8 \mathrm{~nm}) / \mathrm{LiF}(95.5 \mathrm{~nm})$ which improve the device absorption based on a suitable total transmittance, and therefore, the efficiency in the solar cell performance. [18], [19]

\section{Methodology}

As it was mentioned, it will be assessing the use of solar dryer with Integrated Photonic Solar Reflectors and PCMs for Andean Products, Maize and Cedron leaves. In order to achieve this goal, it is suggested to, based on Reyes et al. [6] and Bhardwaj et al. [4] and in the Kant et al. surveys the design and methodology to perform the dehydration process of these items in Cayambe, Provence of Pichincha in Ecuador, which has a solar radiation of around 5.200 $\mathrm{kWh} / \mathrm{m} 2 /$ day. [20] [21] [22]

According Tomar et al. [23] the Initial moisture of Maize is 20 (\% wet basis), and the final moisture after the dry process is $16 \%$, and the maxim allowed temperature for the dehydration process without losing quality is $45^{\circ} \mathrm{C}$. For Cedron Leaves (Aloysia citriodora), as there is no information available, Bhardwaj et al. [4] mentions different medicinal herbs, some of them native from Asia, and, for this case it will be consider the Adhatoda vasica nees, which has an initial moisture of $74 \%$ and the final will be $3 \%$, and the max. allowed temperature is also $45^{\circ} \mathrm{C}$.

In the followed scheme, Fig. 1, it is shown the proposed design of the solar dryer with Integrated Photonic Solar Reflectors and PCMs that considers isolation and a close cycle of air flux. The indirect solar dryer has Integrated Photonic Solar Reflectors in the solar panel, that will receive the solar radiation and it is expected to increase the efficiency of the dryer process. Inside the panel will be cupper tubes that will contain paraffin wax as PCMs due to they are suitable for moderate temperature ranges $\left(40-75^{\circ} \mathrm{C}\right)$ and coupled to this configuration a chamber with plates for the maize and leave samples. [4] [6] 

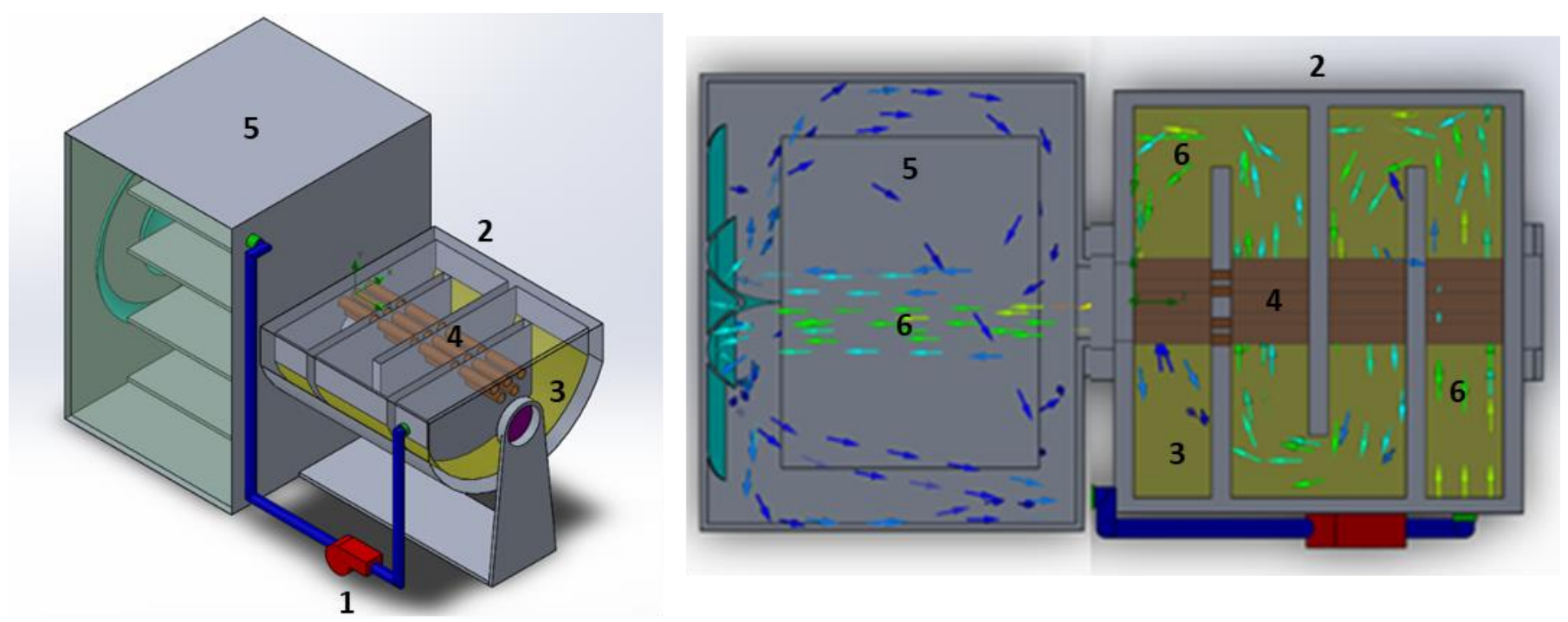

Fig. 1: Proposed design solar dryer+ Integrated Photonic Solar Reflectors and PCMs (Lateral view solar panel and air flow). 1: Blower; 2: Solar Panel, 3: Photonic Solar Reflectors, 4: PCMs, 5: Chamber, 6. Air flux

It will be considered the use of a blower to duct the air through the solar dryer that contains the PCMs in cupper tubes (orange), then, the heated air will flow along the chamber through the food products. This performance will happen during daily hours. By night, the same blower will conduct air to the panel and the PCMs will transfer their heat to this fluid. The resulting heat air will dry the food samples. To guaranty the dehydration process in the stablished temperature conditions of $45{ }^{\circ} \mathrm{C}$ it is expected to add a monitoring system in the chamber that will turn on and off the blower.

As drying models, it will be used the equation for the moisture food products variation in time in the solar system, based on the Bhardwaj et al. [4] research:

$$
\begin{gathered}
\frac{M_{(t)}-M_{(e q)}}{M_{(o)}-M_{(e q)}}=\frac{M_{(t)}}{M_{(o)}} \\
\frac{M_{(t)}}{M_{(o)}}=e^{-k t^{n}}
\end{gathered}
$$

Where:

$M_{(t)}$ : Moisture in a time $\mathrm{t}$

$M_{(e q)}$ : Equilibrium Moisture

$M_{(o)}$ : Moisture in a time t

The shrinkage ratio (SR) will be obtained by the relation between the moisture in time $\mathrm{t}$ and cero, as it follows:

$$
S R=\frac{M_{(t)}}{M_{(o)}}
$$

The dryer thermal efficiency $\eta$ will be defined as the ratio between the heat absorbed $\left(\mathrm{Q}_{\mathrm{Abs}}\right)$ by the drying air from the solar panel and the nominal heat $\left(\mathrm{Q}_{\mathrm{N}}\right)$ necessary to keep the air at a pre-fixed temperature [6] :

$$
\begin{gathered}
Q_{A b s}=m_{h} \cdot C_{s} \cdot\left(T_{\text {out }}-T_{a m b}\right) \\
Q_{N}=m_{h} \cdot C_{s} \cdot\left(T_{f i x}-T_{a m b}\right)
\end{gathered}
$$




$$
\eta=\frac{Q_{A b s}}{Q_{N}}
$$

The heat transfer from the sun to the solar collector considers parameters such as:

A: effective area that receives heat

$$
Q_{R}=A_{c o l} \cdot \cos (\alpha) \cdot \int I_{t} \cdot(d t)
$$

I: Direct normal irradiation

In a first stage, it will be performed the solar dryer without PCM's to evaluate the process of dehydration, and in a second stage, PCMs will be used, to compare and to evaluate solar irradiation in the study area. It is expected to assess the methodology and, as a result, to optimize the solar dryer proposed and chosen for this research.

Taking in consideration the Heat transfer from the PCMs materials to the air to dry the products during the night:

$$
\begin{gathered}
Q_{L}=m \Delta h_{s l} \\
Q_{S}=m C p \Delta T \\
Q_{P C M}=Q_{S s o l}+Q_{L}+Q_{S l i q}
\end{gathered}
$$

Where:

$Q_{L}:$ Latent Heat

$Q_{S}:$ Sensible Heat

$\Delta h s l$ : Enthalpy variation involved in the change of solid-liquid phase

$Q_{S s o l}:$ Sensible Heat in solid phase

$Q_{S l i q}:$ Sensible Heat in liquid phase

The validation of this methodology will be performed using a prototype, in the laboratory. It will be evaluated the solar dryer and the performance of the chosen PCMs materials, by give them a measured electric charge like the quantity of energy that will be received by the solar panels. The results of the dehydration process in the Maize and Cedron leaves will be evaluated. The dissemination of the results will be published and exposed in scientific congresses.

\section{Results}

The expected results are:

- After an evaluation of its performance in the environmental conditions in Cayambe, the selected indirect solar dryer with Integrated Photonic Solar Reflectors, and the organic PCMs for the dehydration process was suggested to be changed focusing in its dimensions.

- According to the data obtained and the survey conducted in another study performed in Chile, the efficiency of the equipment (solar system) is higher in Ecuador due to the use of Integrated Photonic Solar Reflectors which increase the potential of the solar dehydration.

- The estimated time to dry the Maize and the Cedron leaves is reduced, because the irradiation harnessed in the analysed is higher than the ones in the surveys used as base for this study.

- Assessed the use of PCM, as expected, they increase the efficiency of the energy system due to its employment during nights or when the sunlight it is not available.

- The results and findings will be published and exposed in in scientific congresses.

\section{Discussion}

In this study it is shown the performance assessment of a solar dryer with Integrated Photonic Solar Reflectors and the thermal energy storage (PCMs) to dehydrate Andean products, and to evaluate the efficiency of the system in Ecuador and in the Province of Pichincha. 
It is showed that irradiation has a key role in the drying process, and thanks to the use of Integrated Photonic Solar Reflectors and PCMs, it won't be necessary to add extra power sources in order to accomplish the dry process of the food products. It is also important to indicate that due to solar energy available it is possible to modify or reduce the size of the solar dryer that was chosen, and with this, the economic resources for their built or acquisition will be a smaller amount.

\section{Conclusion}

After to perform the research and the validation of the experimentation, the notable findings are consolidated as follows:

- The irradiation that Province of Pichincha has is an important factor of design for the solar dryer, and it will be considered for the building process.

- It will be shown a reduction in the time of drying the Maize and leaves in the solar system located in the Andean Region.

- It will be exposed that the use of Integrated Photonic Solar Reflectors and PCMs increase the thermal efficiency of the solar system and reduce the dehydration time of the selected products.

\section{Acknowledgements}

We would like to specially thank to Escuela Politécnica Nacional and the Project "PIJ 17-09" for the financial aid and cooperation for the development of this Research Project.

\section{References}

[1] FAO, "Food losses and waste in Latin America and the Caribbean," 2014.

[2] D. Stephan Krall, "What is sustainable agriculture?," Sustain. Agric. Res. Educ., 2015.

[3] R. Sanchez and F. Llive, "Panorama Agroeconómico Ecuador 2016," 2016.

[4] A. K. Bhardwaj, R. Chauhan, R. Kumar, M. Sethi, and A. Rana, "Experimental investigation of an indirect solar dryer integrated with phase change material for drying valeriana jatamansi (medicinal herb)," Case Stud. Therm. Eng., vol. 10, no. March, pp. 302-314, 2017.

[5] J. Pereira da Cunha and P. Eames, "Thermal energy storage for low and medium temperature applications using phase change materials - A review," Appl. Energy, vol. 177, pp. 227-238, 2016.

[6] A. Reyes, A. Mahn, and F. Vásquez, "Mushrooms dehydration in a hybrid-solar dryer, using a phase change material," Energy Convers. Manag., vol. 83, pp. 241-248, 2014.

[7] S. M. Shalaby, M. A. Bek, and A. A. El-Sebaii, "Solar dryers with PCM as energy storage medium: A review," Renew. Sustain. Energy Rev., vol. 33, pp. 110-116, 2014.

[8] L. M. Bal, S. Satya, and S. N. Naik, "Solar dryer with thermal energy storage systems for drying agricultural food products: A review," Renew. Sustain. Energy Rev., vol. 14, no. 8, pp. 2298-2314, 2010.

[9] LA-Energy Review, "Ecuador Solar Map - Latin American Energy Review," 2016. [Online]. Available: http://carlosstjames.com/renewable-energy/why-ecuador-doesnt-have-a-renewable-energysector/ecuador-solar-map/. [Accessed: 01-Apr-2018].

[10] Bayer, "El maíz, un alimento fundamental de identidad - Bayer Ecuador," 2017. [Online]. Available: https://www.cropscience.bayer.ec/es-EC/Noticias/Noticias/2017/Septiembre/Maiz-alimentoancestral.aspx. [Accessed: 03-Apr-2018].

[11] "Herbal Monograph: Lemon Verbena."

[12] M. Kumar, S. K. Sansaniwal, and P. Khatak, "Progress in solar dryers for drying various commodities," Renew. Sustain. Energy Rev., vol. 55, pp. 346-360, 2016.

[13] E. K. Akpinar, "Drying of mint leaves in a solar dryer and under open sun: Modelling, performance analyses," Energy Convers. Manag., vol. 51, no. 12, pp. 2407-2418, 2010.

[14] B. Bena and R. J. Fuller, "Natural convection solar dryer with biomass back-up heater," Sol. Energy, vol. 72, no. 1, pp. 75-83, 2002.

[15] A. K. Pandey, M. S. Hossain, V. V. Tyagi, N. Abd Rahim, J. A. L. Selvaraj, and A. Sari, "Novel 
approaches and recent developments on potential applications of phase change materials in solar energy," Renew. Sustain. Energy Rev., vol. 82, no. July 2017, pp. 281-323, 2018.

[16] A. Sharma, V. V. Tyagi, C. R. Chen, and D. Buddhi, "Review on thermal energy storage with phase change materials and applications," Renew. Sustain. Energy Rev., vol. 13, no. 2, pp. 318-345, 2009.

[17] A. Bland, M. Khzouz, T. Statheros, and E. I. Gkanas, "PCMs for residential building applications: A short review focused on disadvantages and proposals for future development," Buildings, vol. 7, no. 3, 2017.

[18] P. Pathi, A. Peer, and R. Biswas, "Nano-photonic structures for light trapping in ultra-thin crystalline silicon solar cells," Nanomaterials, vol. 7, no. 1, pp. 1-16, 2017.

[19] Yu, Wenjuan, Shen, Liang, Shen, Ping Long, Yongbing Sun, Hongwei Chen, Weiyou Ruan, Shengping., "Semitransparent polymer solar cells with 5\% power conversion efficiency using photonic crystal reflector," ACS Appl. Mater. Interfaces, vol. 6, no. 1, pp. 599-605, 2014.

[20] K. Kant, A. Shukla, A. Sharma, A. Kumar, and A. Jain, "Thermal energy storage based solar drying systems: A review," Innov. Food Sci. Emerg. Technol., vol. 34, pp. 86-99, 2016.

[21] G. Akmak and C. Yildiz, "The drying kinetics of seeded grape in solar dryer with PCM-based solar integrated collector," Food Bioprod. Process., vol. 89, no. 2, pp. 103-108, 2011.

[22] L. M. Bal, S. Satya, S. N. Naik, and V. Meda, "Review of solar dryers with latent heat storage systems for agricultural products," Renew. Sustain. Energy Rev., vol. 15, no. 1, pp. 876-880, 2011.

[23] V. Tomar, G. N. Tiwari, and B. Norton, "Solar dryers for tropical food preservation: Thermophysics of crops, systems and components," Sol. Energy, vol. 154, pp. 2-13, 2017. 